\title{
Laser fluence dependence of periodic grating structures formed on metal surfaces under femtosecond laser pulse irradiation
}

\section{$\operatorname{AUTHOR}(\mathrm{S}):$}

Okamuro, Kiminori; Hashida, Masaki; Miyasaka, Yasuhiro; Ikuta, Yoshinobu; Tokita, Shigeki; Sakabe, Shuji

\section{CITATION:}

Okamuro, Kiminori ...[et al]. Laser fluence dependence of periodic grating structures formed on metal surfaces under femtosecond laser pulse irradiation. Physical Review B 2010, 82(16): 165417.

\section{ISSUE DATE:}

2010-10

URL:

http://hdl.handle.net/2433/145965

RIGHT:

(C) 2010 The American Physical Society. 


\title{
Laser fluence dependence of periodic grating structures formed on metal surfaces under femtosecond laser pulse irradiation
}

\author{
Kiminori Okamuro, Masaki Hashida, * Yasuhiro Miyasaka, Yoshinobu Ikuta, Shigeki Tokita, and Shuji Sakabe \\ Advanced Research Center for Beam Science, Institute for Chemical Research, Kyoto University, Gokasho, Uji, Kyoto 611-0011, Japan \\ and Department of Physics, Graduate School of Science, Kyoto University, Kitashirakawa, Sakyo, Kyoto 606-8502, Japan
}

(Received 2 June 2010; published 8 October 2010)

\begin{abstract}
Periodic structures self-formed on the surface of several metals under femtosecond laser-pulse irradiation are investigated by electron microscopy. For the self-formation of periodic gratings on metal surfaces, the interspaces of the periodic structures depend on laser fluence. This dependence is the same for all metals, although the range of laser fluence in which the structures are formed differs between metals. The laser fluence dependence can be explained by the generation of a plasma wave through the parametric decay of laser light [S. Sakabe, M. Hashida, S. Tokita, S. Namba, and K. Okamuro, Phys. Rev. B 79, 033409 (2009)]. This indicates that the formation of periodic structures depends not only on metal properties but also on the electron density of plasma produced on a surface by femtosecond laser pulses.
\end{abstract}

DOI: 10.1103/PhysRevB.82.165417

PACS number(s): 79.70.+q, 79.20.Ds, 78.20.Jq

\section{INTRODUCTION}

Periodic grating structures self-formed inside or on the surface of materials under femtosecond-pulse laser irradiation have been found for insulators, ${ }^{1}$ semiconductors, ${ }^{2}$ and metals. $^{3}$ These structures have certain features that are not observed for picosecond- or nanosecond-pulse lasers. For transparent materials, self-formed grating structures oriented perpendicular to the laser polarization direction are attributed to the following mechanisms: laser-induced plasma waves in bulk plasma for glasses, ${ }^{4,5}$ the excitation of surface-plasmon polaritons in the surface layer for diamondlike carbon, ${ }^{6}$ and directional atomic surface diffusion anisotropy arising from plasmon-coupled metallic colloid arrays induced by laser pulses for $\mathrm{CaF}_{2}{ }^{7}$ In these reports, the grating spaces depended on laser fluence and the observed discrete spacings (bifurcation) were discussed. The spacings of grating structures for transparent materials are much shorter than the spacings for metals; therefore, the mechanisms proposed for transparent materials cannot be applied to metals.

For metals irradiated with linearly polarized laser light, the grating structures formed on the metal surface are oriented perpendicular to the laser polarization. The interspaces of the grating structures are shorter than the laser wavelength and depend on laser fluence. ${ }^{3-16}$ However, the mechanism of self-formation is still under investigation. The structures depend on material properties and laser parameters (fluency, pulse duration, number of pulses, etc.). Even for the same metal, not all experimental results reported in the literature $^{3-31}$ can be directly compared because of differing parameters, such as the laser parameters. The relation between the type of metal and the interspaces of periodic grating structures has not been investigated, and therefore the amount of systematic experimental data is insufficient for discussing the mechanism at present.

We have reported that the formation of grating interspaces depends on laser fluence in the case of copper ${ }^{3}$ with $\leq 100$ fs laser pulses. At laser fluence near the ablation threshold, the grating structures have an interspace of $300 \mathrm{~nm}$, which is much shorter than the laser wavelength of $800 \mathrm{~nm}$. The in- terspace increases up to about $700 \mathrm{~nm}$ as laser fluence increases. This dependence of the interspaces on laser fluence has been explained by Sakabe et al. on the basis of the parametric decay (stimulated Raman scattering) model. ${ }^{32}$ In this model, a surface-plasma waves are induced at the interface between free space (air) and either laser-produced plasma or metal plasma by parametric decay; in other words, the incident laser light decays into a surface-plasma wave and a scattered electromagnetic wave. Although the process whereby generation of the surface-plasma wave leads to formation of periodic structures is not yet fully understood, we assume the following: when the plasma wave travels slowly, locally charged areas are periodically generated on the surface and positively charged areas are exploded toward free space by the Coulomb repulsive force (Coulomb explosion); consequently, a thin layer is ablated and the grating pattern is
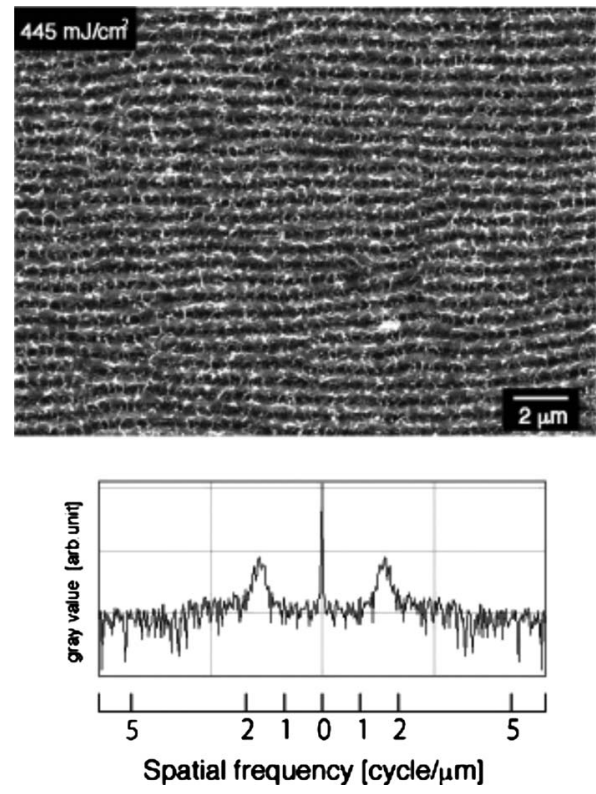

FIG. 1. SEM images of periodic surface structures on tungsten produced by 50 laser pulses of 160 fs at laser fluence of $445 \mathrm{~mJ} / \mathrm{cm}^{2}$ and the Fourier-transform spectrum. 

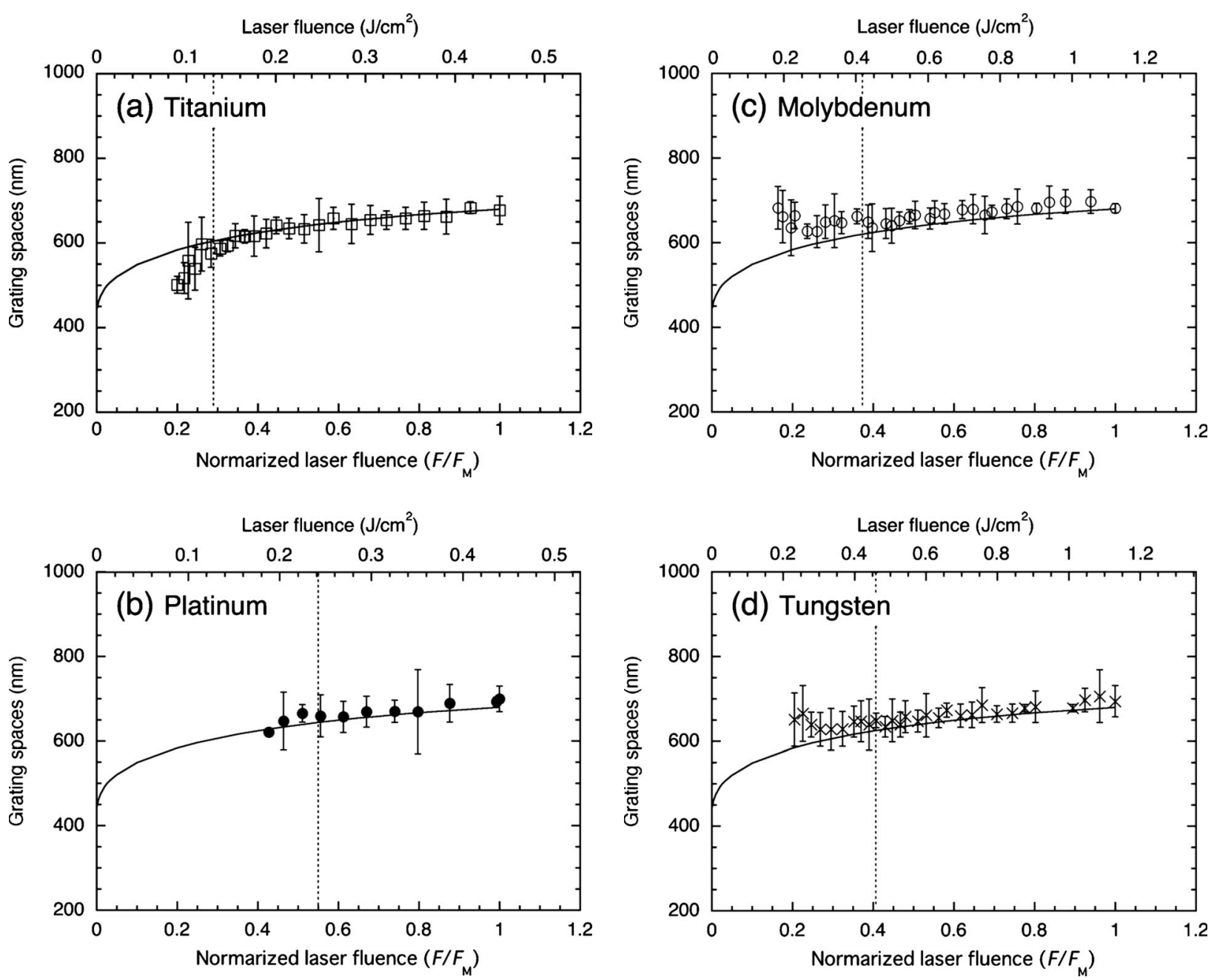

FIG. 2. Laser fluence dependence of the periodic structure interspaces produced by femtosecond laser pulses for (a) Ti, (b) Pt, (c)Mo, and (d) W (pulse duration: $160 \mathrm{fs}$ ). The interspace of the grating structure was determined by analyzing a set of ten irradiated spots on the metal surface. Error bars show the standard deviation of the interspaces. Laser fluence is normalized by the upper limit on laser fluence for producing periodic structures, $F_{M}$. Solid lines show calculation results according to the parametric decay model proposed by Sakabe $e t$ al. (Ref. 32), and dotted lines show the laser fluence for the ablation rate of $10 \mathrm{~nm} / \mathrm{pulse}$.

printed. ${ }^{33}$ The wave number (wavelength) of the surfaceplasma wave induced on the surface depends on only the plasma frequency (electron density) of the surface plasma (see Fig. 3 of Ref. 32). The ratio of the wavelength of the plasma wave to the wavelength of the laser light changes from 0.5 to 0.85 for plasma frequencies in the range of 0 $<\omega_{p} / \sqrt{2}<\omega_{L}$, where $\omega_{p}$ and $\omega_{L}$ are the frequencies of the plasma and laser light, respectively. When the plasma electron density depends on laser fluence, the wavelength of the plasma wave in turn depends on laser fluence. The results reported in Ref. 3 for copper are well explained by this model; however, experimental data are too scarce at present for this model to be confirmed.

In the present study, we investigated by electron microscopy the state of surfaces irradiated with femtosecond laser pulses for $\mathrm{Al}, \mathrm{Au}, \mathrm{Cu}, \mathrm{Ti}, \mathrm{Pt}, \mathrm{Mo}$, and $\mathrm{W}$ metals. In addition, we evaluated the dependence of periodic structure interspaces on laser fluence to discuss the validity of the parametric decay model.

\section{EXPERIMENTAL}

To ablate metals, we used linearly polarized laser pulses (160 fs pulse duration, $800 \mathrm{~nm}$ center wavelength, $10 \mathrm{~Hz}$ repetition rate) from a Ti:sapphire chirped-pulse amplification laser system ( $\mathrm{T}^{6}$-laser, ICR, Kyoto University). The root-mean-square fluctuation of pulse energy was $\pm 0.3 \%{ }^{34}$ In air, the laser pulses were focused onto the metals by a spherical lens with focal length of $100 \mathrm{~mm}(F / 15)$. The focal spot was $45 \mu \mathrm{m}$ in diameter. To avoid nonuniformity of structure in the irradiated area on the surface, the laser intensity distribution was adjusted to be spatially uniform. A super-Gaussian profile was produced as follows. From a Gaussian profile pulse, the center part was cut away by an aperture of $1 \mathrm{~mm}$ in diameter, and the cut image was relayed to the target surfaces. The laser pulses were directed at normal incidence onto the target surface. The targets were Al, $\mathrm{Au}, \mathrm{Cu}, \mathrm{Ti}, \mathrm{Pt}, \mathrm{Mo}$, and $\mathrm{W}$ metals, which had been mechanically polished. The roughness, as characterized by the arith- 
TABLE I. Upper limit on laser fluence for producing periodic structures, $F_{M}$, and the laser fluence corresponding to an ablation rate of $10 \mathrm{~nm} /$ pulse, $F_{10 \mathrm{~nm}}$, are listed for the metals. $T_{m}$ is the melting point.

\begin{tabular}{lccc}
\hline \hline Metal & $\begin{array}{c}T_{\text {melt }} \\
(\mathrm{K})\end{array}$ & $\begin{array}{c}F_{M} \\
\left(\mathrm{~J} / \mathrm{cm}^{2}\right)\end{array}$ & $\begin{array}{c}F_{10 \mathrm{~nm}} \\
\left(\mathrm{~J} / \mathrm{cm}^{2}\right)\end{array}$ \\
\hline $\mathrm{Al}$ & 933 & & \\
$\mathrm{Au}$ & 1337 & & \\
$\mathrm{Cu}$ & 1356 & & \\
$\mathrm{Ti}$ & 1938 & 0.45 & 0.13 \\
$\mathrm{Pt}$ & 2042 & 0.44 & 0.24 \\
$\mathrm{Mo}$ & 2888 & 1.1 & 0.42 \\
$\mathrm{~W}$ & 3672 & 1.1 & 0.46 \\
\hline \hline
\end{tabular}

metic mean value, was less than $2 \mathrm{~nm}$ for all metals. Through a pair of half-wave plates and two polarizers, the energy was varied in the range of $1.1-34 \mu \mathrm{J}$, corresponding to fluence of $50-2100 \mathrm{~mJ} / \mathrm{cm}^{2}$. Energy (average of 500 pulses) was measured just before irradiating a target for each laser fluence. The number of irradiating pulses was 50 in all experiments. The irradiated spots were separated by more than $100 \mu \mathrm{m}$. Laser-produced surface structures were examined by scanning electron microscopy (SEM) (JSM-5560 made in JEOL). The periodic grating interspace was determined by reading the peak value in the frequency domain after taking the Fourier transform for the $20 \mu \mathrm{m} \times 15 \mu \mathrm{m}$ area of the SEM image, which is equivalent to the laser irradiated area on the targets. The resolution of the present measurements of the periodic spacing was better than $34 \mathrm{~nm}$. The interspace of the grating structure was obtained by analyzing a set of ten irradiated spots on a metal surface. Figure 1 shows a typical SEM image and the corresponding Fourier-transform spectrum. To evaluate the ablation rate, the crater produced by 200 laser pulses was measured by confocal laser scanning microscopy (HL-150, Lasertec).

\section{RESULTS AND DISCUSSION}

Self-formation of periodic structures was not observed for $\mathrm{Al}, \mathrm{Au}$, and $\mathrm{Cu}$ metals but was observed for Ti, Pt, Mo, and $\mathrm{W}$ metals. Here, we must make particular note of $\mathrm{Cu}$ metal. As reported in Ref. 3, periodic structures clearly formed on $\mathrm{Cu}$, and the interspaces depended on laser fluence. In the present experiments, self-formation of periodic structures was not observed for $\mathrm{Cu}$. We discuss these findings later. Figure 2 shows the dependence of the periodic structure interspaces on laser fluence for Ti, Pt, Mo, and W metals. Al, $\mathrm{Au}$, and $\mathrm{Cu}$ are excluded from the following discussion as no periodic structures were observed for these metals. The upper limit on the laser fluence at which the periodic structures are formed can be seen; let us define this fluence as $F_{M}$. The laser fluence in Fig. 2 is normalized by the fluence $F_{M}$ (see Table I for $F_{M}$ of each metal). As the laser fluence increases, the periodic interspace increases up to about $700 \mathrm{~nm}$ for all metals, and this maximum interspace is obtained at the fluence $F_{M}$. Let us compare these experimental results with the

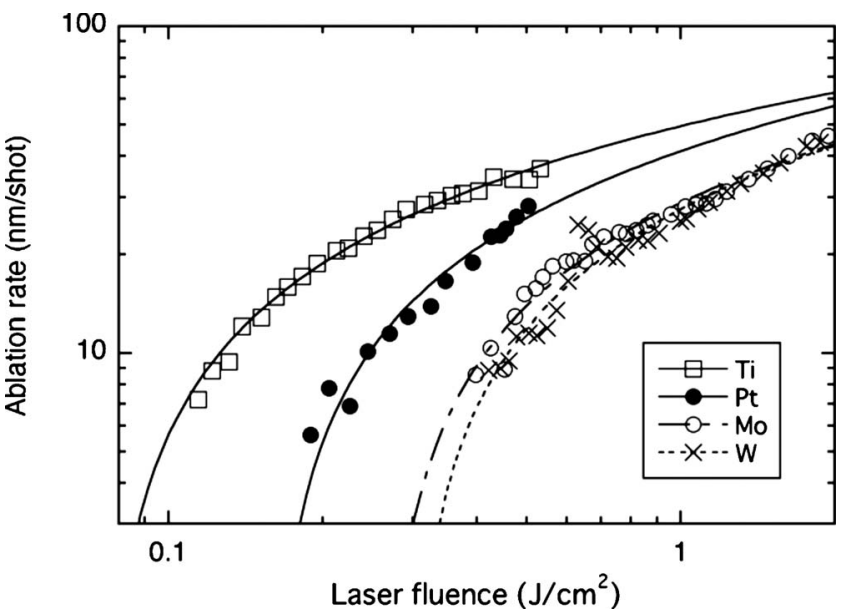

FIG. 3. Laser fluence dependence of ablation rate for metals on the surface of which self-formation of periodic structures occurs. Experimental data were fit to the well-known function $L$ $=\alpha^{-1} \ln \left(F_{L} / F_{t h}\right)$, where $\alpha^{-1}$ is the optical absorption or heat penetration coefficient, $F_{L}$ is the laser fluence, and $F_{t h}$ is the ablation threshold fluence.

predictions from the model of plasma wave induction by parametric decay of laser light. ${ }^{32}$ Here, the model is briefly described. The parametric process of photon $\rightarrow$ photon + plasmon can occur on a plasma surface as well as in a bulk plasma (i.e., stimulated Raman scattering). The parametric conditions of $\omega_{L}=\omega_{2}+\omega_{\text {SP }}$ and $\mathbf{k}_{L}=\mathbf{k}_{\mathbf{2}}+\mathbf{k}_{\mathrm{SP}}$, where the subscripts $L, 2$, and SP indicate the incident laser light, scattered light, and surface-plasma wave, respectively, are reduced to

$$
\begin{gathered}
\omega_{L}-\omega_{\mathrm{SP}}=c k_{\mathrm{SP}}-c k_{L}, \quad \omega_{L}=c k_{L}, \\
\omega_{\mathrm{SP}}^{2}=c^{2} k_{\mathrm{SP}}^{2}+\frac{1}{2} \omega_{P}^{2}-\sqrt{c^{4} k_{\mathrm{SP}}^{4}+\frac{1}{4} \omega_{P}^{4}}
\end{gathered}
$$

The wave number of the plasma wave induced by the parametric process can be related to the plasma frequency, and the $k_{L} / k_{\mathrm{SP}}$ ratio $\left(=\lambda_{\mathrm{SP}} / \lambda_{L} ; \lambda\right.$ is the wavelength $)$ changes from 0.5 to 0.85 for plasma frequencies in the range of 0 $<\omega_{p} / \sqrt{2}<\omega_{L}$, where the plasma wave number increases as the plasma frequency decreases. As mentioned above, assuming that the self-formation is induced by the plasma wave, the grating spaces correspond to the wavelength of the induced plasma wave, and the fluence dependence of the interspaces can be reduced to plasma density dependence. The dependence of the surface electron density $n_{\mathrm{es}}$ on the laser fluence $F_{L}$ can be interpreted as follows. The electron density $n_{e}$ of the plasma bulk produced on the surface by the laser is proportional to the laser energy: $n_{e} \propto F_{L}$. The heated plasma bulk with temperature $T_{e}$ expands at the sonic speed $c_{s}=\sqrt{k_{B} T_{e} / m}$, and the surface electron density decreases from the bulk density as $n_{e} / c_{s}$, and the temperature is proportional to the laser energy: $T_{e} \propto F_{L}$. Therefore, the surface electron density is related to the laser fluence as $n_{\mathrm{es}} \propto n_{e} / c_{s} \propto n_{e} / \sqrt{T}$ $\propto \sqrt{F_{L}}$. It is reasonable to assume that the plasma frequency is $\omega_{p}=\sqrt{2} \omega_{L}$ for the laser fluence $F_{M}$ since no grating structures are produced at laser fluence over $F_{M}$. In this case, 
$n_{\mathrm{es}}\left(\mathrm{cm}^{-3}\right)=3.5 \times 10^{21} \sqrt{\left(F_{L} / F_{M}\right)}$. Applying this expression together with $\omega_{p}=\sqrt{4 \pi n_{\mathrm{es}} e^{2} / m}$ to the dependence of $\lambda_{\mathrm{SP}} / \lambda_{L}$ on $\omega_{p}$, the spatial dependence of the laser fluence is obtained. This relation is shown as a solid line in Fig. 2. For each metal, the experimental results agree reasonably well with this model, although there is disagreement in the relatively low fluence range. This disagreement is considered to arise from nonuniformity of ablation. At laser fluence near the ablation threshold, the amount of ablated plasma is too small to construct a uniform plasma surface. The measured ablation rate is shown in Fig. 3. The threshold of nanostructure formation can be defined for an ablation rate less than several nanometers per pulse. For such a thin ablated layer, a uniform plasma surface is difficult to form. Here, let us define $F_{10 \mathrm{~nm}}$ as the fluence at which a $10 \mathrm{~nm}$ layer is ablated. The values of $F_{10 \mathrm{~nm}}$ are listed in Table I and are shown in Fig. 2 as dotted lines. As this figure shows, the model is in fairly good agreement with the experimental results in the fluence range of $F_{10 \mathrm{~nm}}<F_{L}<F_{M}$, and a common fluence dependence of the periodic grating interspaces can be found for self-formation induced by femtosecond laser pulses for all metals. These experimental results indicate that the interspaces of the self-formed periodic structure depend not on metal characteristics but rather on the density of the surface plasma produced by a laser pulse. For high laser fluence $\left(F_{L}>F_{M}\right)$, the plasma expands too greatly for a clear surface to be constructed, or the plasma density is too high; in such cases, a surface-plasma wave is not produced.

Now, let us return our attention to the metals for which self-formation of periodic structures was not observed. Metals such as $\mathrm{Al}$ and $\mathrm{Au}$, which have low melting points, easily form gas plasmas, and thus $F_{M}$ is not defined. For $\mathrm{Cu}$ metal, which melts at an intermediate temperature, $F_{M}$ is defined for $100 \mathrm{fs}$ pulses, but not for $160 \mathrm{fs}$ pulses, because the plasma expands too greatly and a clear surface is not produced for the longer pulse. Here, we discuss the reason why selfformation of periodic structures was not restored in the case of high fluence for the $160 \mathrm{fs}$ pulse. The experimental results show that the periodic nanostructures were observed in the laser fluence range from $\sim F_{10 \mathrm{~nm}}$ to $F_{M}$. Therefore, the condition of nanostructures formation should be satisfied the following $\sim F_{10 \mathrm{~nm}} \leq F_{M}$. The ablation threshold fluence, $F_{t h}$, for metals is strongly dependent on laser pulse duration, $\tau_{L}$, as $F_{t h} \propto \tau_{L}^{1 / 2}$ in the laser fluence range of interest. This tendency has been reported previously. ${ }^{3,35}$ Therefore, the laser fluence corresponding to an ablation rate of $10 \mathrm{~nm} / \mathrm{pulse}$, $F_{10 \mathrm{~nm}}$, also increases with increasing laser pulse duration. For pulse duration of $160 \mathrm{fs}, F_{10} \mathrm{~nm}$ was $0.55 \mathrm{~J} / \mathrm{cm}^{2}$, which is approximately 1.3 times higher than $F_{10 \mathrm{~nm}}$ for pulse duration of $100 \mathrm{fs}$. On the other hand, the upper limit of laser fluence for producing periodic structures, $F_{M}$, decreases with decreasing melting temperature of the metals. For $\mathrm{Cu}$ irradiated with $160 \mathrm{fs}$ pulses, $F_{M}$ is expected to be equal to or less than $\sim F_{10 \mathrm{~nm}}$. Therefore, the conditions for nanostructure formation on $\mathrm{Cu}$ are not satisfied in the case of longer pulse duration.

\section{CONCLUSION}

In summary, we measured the periodic structures selfformed on the surface of $\mathrm{Al}, \mathrm{Au}, \mathrm{Cu}, \mathrm{Ti}, \mathrm{Pt}, \mathrm{Mo}$, and $\mathrm{W}$ metals. We found that the laser fluence dependence of periodic structure interspaces is the same for all metals on which the structure is self-formed. The dependence can be explained by the induction of a surface-plasma wave through the parametric decay of laser light, and can be reduced to the dependence of the density of laser-produced plasmas.

\section{ACKNOWLEDGMENTS}

We thank T. Kanaya, K. Nishida, and T. Terashima for their assistance with SEM and AFM. This research was financially supported by a Grant-in-Aid for the Global COE Program "The Next Generation of Physics, Spun from Universality and Emergence" from the Ministry of Education, Culture, Sports, Science and Technology (MEXT) of Japan and supported in part by the Amada Foundation for Metal Work Technology and a Grant-in Aid for Scientific Research (C) (Grant No. 22560720) from MEXT, Japan.

\footnotetext{
*Corresponding author.

${ }^{1}$ F. Costache, M. Henyk, and J. Reif, Appl. Surf. Sci. 208-209, 486 (2003).

${ }^{2}$ N. Yasumaru, K. Miyazaki, and J. Kiuchi, Appl. Phys. A: Mater. Sci. Process. 76, 983 (2003).

${ }^{3}$ M. Hashida, M. Fujita, Y. Izawa, and A. F. Semerok, in Laser Precision Microfabrication, Proceedings of SPIE Vol. 4830, edited by I. Miyamoto, K. F. Kobayashi, K. Sugioka, R. Poprawe, and H. Helvajian (SPIE, Washington, 2002), pp. 452-457.

${ }^{4}$ J. Reif, O. Varlamova, and F. Costache, Appl. Phys. A: Mater. Sci. Process. 92, 1019 (2008).

${ }^{5}$ F. Costache, S. Eckert, and J. Reif, Appl. Phys. A: Mater. Sci. Process. 92, 897 (2008).

${ }^{6}$ G. Miyaji and K. Miyazaki, Opt. Express 16, 16265 (2008).

${ }^{7}$ Y. Shimotsuma, P. G. Kazansky, J. Qiu, and K. Hirao, Phys. Rev. Lett. 91, 247405 (2003).
}

${ }^{8}$ A. Y. Vorobyev, V. S. Makin, and C. Guo, J. Appl. Phys. 101, 034903 (2007).

${ }^{9}$ A. Y. Vorobyev and C. Guo, Appl. Surf. Sci. 253, 7272 (2007).

${ }^{10}$ M. Tsukamoto, K. Asuka, H. Nakano, M. Hashida, M. Katto, N. Abe, and M. Fujita, Vacuum 80, 1346 (2006).

${ }^{11}$ V. S. Makin, R. S. Markin, A. Ya Vorobyev, and C. Guo, Tech. Phys. Lett. 34, 387 (2008).

${ }^{12}$ A. Y. Vorobyev and C. Guo, J. Appl. Phys. 104, 063523 (2008).

${ }^{13}$ Q. Z. Zhao, S. Malzer, and L. J. Wang, Opt. Lett. 32, 1932 (2007).

${ }^{14}$ E. V. Golosov, V. I. Emel'yanov, A. A. Ionin, Yu. R. Kolobov, S. I. Kudryashov, A. E. Ligachev, Yu. N. Novoselov, L. V. Seleznev, and D. V. Sinitsyn, JETP Lett. 90, 107 (2009).

${ }^{15}$ J. Wang and C. Guo, J. Appl. Phys. 100, 023511 (2006).

${ }^{16}$ G. A. Shafeev, J. Degert, N. Lascoux, and E. Freysz, Proceedings of the Conference on lasers and Electro-Optics (CLEO), 
Baltimore, Maryland, 2005 (unpublished).

${ }^{17}$ A. Y. Vorobyev and C. Guo, J. Appl. Phys. 103, 043513 (2008).

${ }^{18}$ J. Wang and C. Guo, Appl. Phys. Lett. 87, 251914 (2005).

${ }^{19}$ J. Gottmann, D. Wortmann, and M. H-Jungemann, Appl. Surf. Sci. 255, 5641 (2009).

${ }^{20}$ A. Y. Vorobyev and C. Guo, Phys. Rev. B 72, 195422 (2005).

${ }^{21}$ J. Kim, S. Na, S. Cho, W. Chang, and K. Whang, Opt. Lasers Eng. 46, 306 (2008).

${ }^{22}$ S. E. Kirkwood, A. C. van Popta, Y. Y. Tsui, and R. Fedosejevs, Appl. Phys. A: Mater. Sci. Process. 81, 729 (2005).

${ }^{23}$ A. Weck, T. H. R. Crawford, D. S. Wilkinson, H. K. Haugen, and J. S. Preston, Appl. Phys. A: Mater. Sci. Process. 89, 1001 (2007).

${ }^{24}$ A. Weck, T. H. R. Crawford, D. S. Wilkinson, H. K. Haugen, and J. S. Preston, Appl. Phys. A: Mater. Sci. Process. 90, 537 (2008).

${ }^{25}$ R. V. Volkov, D. M. Golishnikov, V. M. Gordienko, and A. B. Savel'ev, JETP Lett. 77, 473 (2003).

${ }^{26}$ A. Y. Vorobyev and C. Guo, Appl. Phys. A: Mater. Sci. Process.
86, 321 (2007).

${ }^{27}$ B. K. Nayak, M. C. Gupta, and K. W. Kolasinski, Appl. Phys. A: Mater. Sci. Process. 90, 399 (2008).

${ }^{28}$ V. Oliveira, S. Ausset, and R. Vilar, Appl. Surf. Sci. 255, 7556 (2009).

${ }^{29}$ Y. Huang, S. Liu, W. Li, Y. Liu, and W. Yang, Opt. Express 17, 20756 (2009).

${ }^{30}$ H. Zhang, M. Tang, J. McCoy, and T. Her, Opt. Express 15, 5937 (2007).

${ }^{31}$ Q. Z. Zhao, S. Malzer, and L. J. Wang, Opt. Express 15, 15741 (2007).

${ }^{32}$ S. Sakabe, M. Hashida, S. Tokita, S. Namba, and K. Okamuro, Phys. Rev. B 79, 033409 (2009).

${ }^{33}$ M. Hashida, S. Namba, K. Okamuro, S. Tokita, and S. Sakabe, Phys. Rev. B 81, 115442 (2010).

${ }^{34}$ S. Tokita, M. Hashida, S. Masuno, S. Namba, and S. Sakabe, Opt. Express 16, 14875 (2008).

${ }^{35}$ M. Hashida, A. F. Semerok, O. Gobert, G. Petite, Y. Izawa, and J. F-. Wagner, Appl. Surf. Sci. 197-198, 862 (2002). 